Document downloaded from:

http://hdl.handle.net/10251/64737

This paper must be cited as:

Balador, A.; Böhm, A.; Uhlemann, E.; Tavares De Araujo Cesariny Calafate, CM.; Cano Escribá, JC. (2015). A reliable token-based MAC protocol for delay sensitive platooning applications. 82nd IEEE Vehicular Technology Conference: VTC2015-Fall. IEEE. doi:10.1109/VTCFall.2015.7390813.

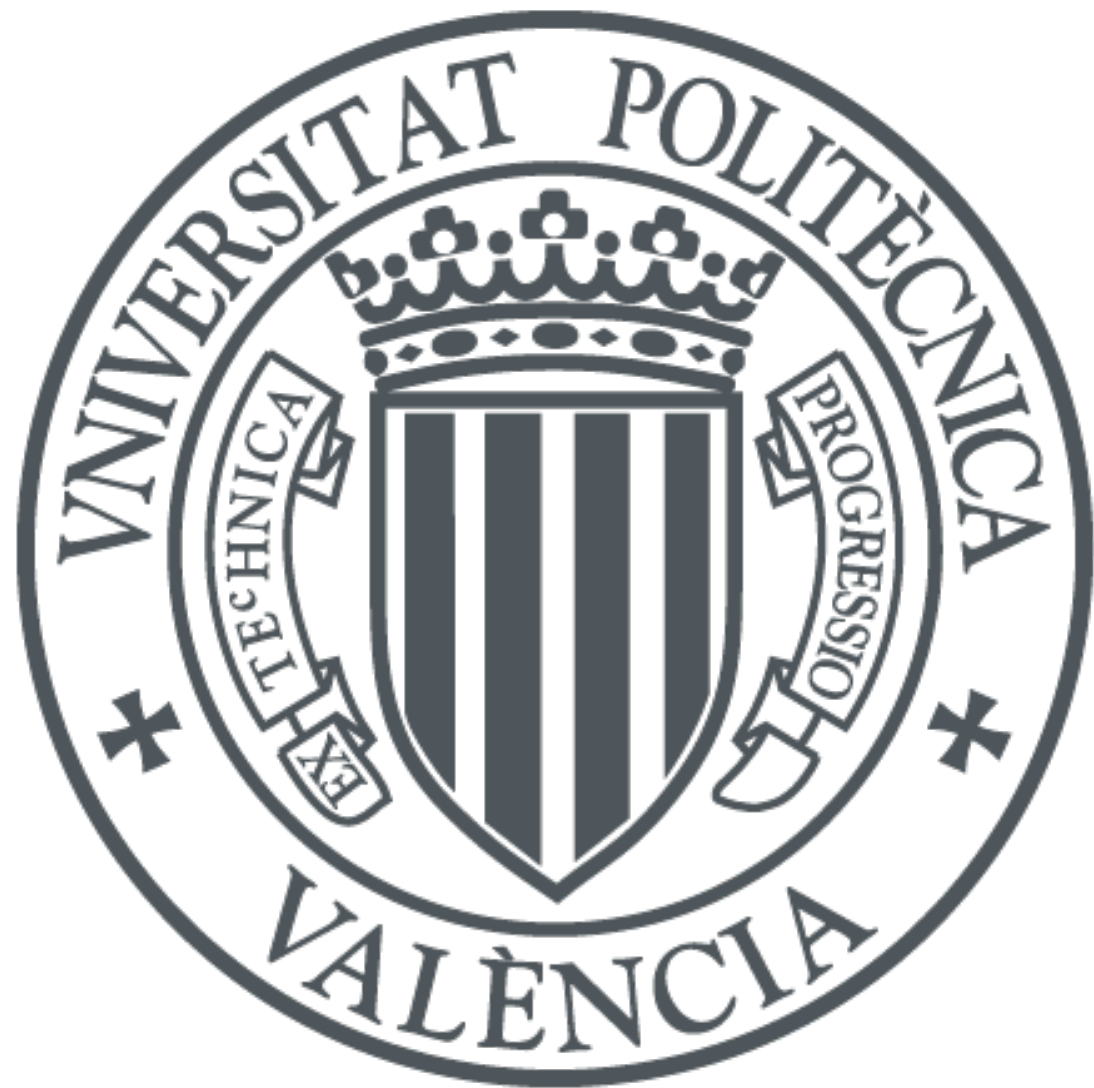

The final publication is available at

http://dx.doi.org/10.1109/VTCFall.2015.7390813 Publisher:

Copyright IEEE

Additional Information

(C) 2015 IEEE. Personal use of this material is permitted. Permission from IEEE must be obtained for all other uses, in any current or future media, including reprinting/republishing this material for advertising or promotional purposes, creating new collective works, for resale or redistribution to servers or lists, or reuse of any copyrighted component of this work in other works. 


\title{
A Reliable Token-Based MAC Protocol for Delay Sensitive Platooning Applications
}

\author{
Ali Balador ${ }^{1,2}$, Annette Böhm ${ }^{2}$, Elisabeth Uhlemann ${ }^{2,3}$, Carlos T. Calafate ${ }^{1}$, and Juan-Carlos Cano ${ }^{1}$ \\ ${ }^{1}$ Universitat Politecnica de Valencia, Spain, alba6@upv.es, \{calafate, jucano\}@disca.upv.es \\ ${ }^{2}$ Halmstad University, Sweden, annette.bohm@hh.se \\ ${ }^{3}$ Mälardalen University, Sweden, elisabeth.uhlemann@mdh.se
}

\begin{abstract}
Platooning is both a challenging and rewarding application. Challenging since strict timing and reliability requirements are imposed by the distributed control system required to operate the platoon. Rewarding since considerable fuel reductions are possible. As platooning takes place in a vehicular ad hoc network, the use of IEEE 802.11p is close to mandatory. However, the 802.11p medium access method suffers from packet collisions and random delays. Most ongoing research suggests using TDMA on top of 802.11p as a potential remedy. However, TDMA requires synchronization and is not very flexible if the beacon frequency needs to be updated, the number of platoon member changes or if retransmissions for increased reliability are required. We therefore suggest a token-passing medium access method where the next token holder is selected based on beacon data age. This has the advantage of allowing beacons to be re-broadcasted in each beacon interval whenever time and bandwidth is available. We show that our token-based method is able to reduce the data age and increase reliability considerably compared to pure 802.11p.
\end{abstract}

\section{INTRODUCTION}

Numerous research efforts have been directed towards Vehicular Ad Hoc Networks (VANETs) since the eighties, but Cooperative Intelligent Transport Systems (C-ITS) and its implementation on the roads is still in its infancy. One of the topics within C-ITS which currently attracts a huge interest is platooning, partly since it has the potential to be one of the first applications to actually be implemented on our roads in the near future. A platoon includes a leading vehicle which is operated by a professional driver, and one or more other vehicles, which are autonomously driven, following the leader in close proximity. Several studies have shown considerable reductions in fuel consumption for platooning scenarios. In [1], Bonnet and Fritz could show a $21 \%$ fuel reduction for trailing trucks travelling at $80 \mathrm{~km} / \mathrm{h}$ with an inter-vehicle gap of 10 m. Even the lead truck showed a fuel reduction of $6 \%$. With heavy vehicles accounting for 5\% of global carbon emissions, there is a clear environmental and economical incentive for transport industries to implement platooning.

Each vehicle must be aware of the position, status and intention of its surrounding vehicles through message broadcasting. For this purpose, two types of messages are typically used: periodic beacons and event-driven messages. Beacons include information such as geographical location, speed, and acceleration, and are only sent to a close neighborhood, as the validity of the information they contain is very limited in time. A large variety of C-ITS based safety applications are built upon the periodic exchange of beacons and their timely and reliable transmission is vital as a vehicle that continuously fails to deliver its beacon becomes invisible to its neighbors resulting in potential hazardous situations. Eventdriven messages are only generated when an event of common interest occurs and spread within an area of interest for the duration of the event.

Most VANET applications use IEEE 802.11p, which is a protocol suite directly based on the random medium access control (MAC) method known as the Carrier Sense Multiple Access (CSMA) algorithm. As shown in several papers published in the area, e.g. [2], [3], [4], CSMA is not able to provide guaranteed delay bounds and sufficient reliability for vehicular scenarios, especially under high channel load. This problem is particularly serious when implementing a (semi)automated driving application such as platooning, where intervehicle spacing is drastically reduced and the control loop that manages and maintains the platoon requires the frequent, timely and reliable exchange of status information (beacons).

Both in the US and in Europe, one dedicated control channel in the $5.9 \mathrm{GHz}$ band is defined, mainly intended for data exchange from traffic-safety applications, e.g., beacons, event-driven messages and service announcements. Additional service channels are available and can, e.g., be dedicated to certain applications as platooning, as long as mandatory listening periods to the control channel are kept. Alternatively, a second transceiver needs to be installed and tuned to the service channel. Platooning application will likely need a higher periodicity compared to the one used in regular VANETs (20 $\mathrm{ms}$ is often mentioned as a realistic update period by truck manufacturers) [5]. Due to current restrictions of beacon frequency on the control channel for VANETs, the issue of interference with other C-ITS based applications, we argue that the use of a separate service channel for platooning is a requirement similarly to what was concluded in [4]. Since heavy vehicles benefit greatly from platooning in terms of reduced fuel consumption, the benefits outweigh the extra cost of an additional transceiver.

Beacons carry highly time-critical information and a missed beacon from another platoon member changes the view a vehicle has of the status of the platoon. Several consecutive beacon misses may force this vehicle to take actions that are both unnecessary and counterproductive to the efficient and safe control of the platoon. The age of the information each platoon member holds about its neighbors is consequently a 
vital parameter to the successful implementation of platooning. Addressing the shortcomings of IEEE 802.11p, while still maintaining the flexibility of a distributed MAC scheme, we propose a token passing MAC method where the next token holder is selected based on the data age of its previously received beacons. The token is piggybacked on the beacon to avoid additional control traffic. Whenever a platoon member is given the token, it will transmit its beacon as soon as the channel is sensed free, while at the same time passing the token to the next token holder. This allows beacons to be rebroadcasted one or several times during a beacon interval as long as time and bandwidth is available, thereby increasing the probability of packet reception by the other platoon members. Note that our scheme can be implemented on top of IEEE 802.11p, and thus no changes to IEEE 802.11p compliant hardware are required.

The rest of the paper is organized as follows: Section II outlines the related work on beacon broadcasting and platooning. Section III details our proposed MAC method. Simulation details are then presented in Section IV, while the simulation results are shown in Section V. Finally, section VI concludes this paper.

\section{BACKGROUND AND RELATED WORKS}

Recently, IEEE 802.11p, an amendment to the IEEE 802.11 standard for inter-vehicle communications, was approved [6]. IEEE 802.11 p defines specifications for the physical and MAC layers. Despite the built-in mechanisms of the CSMA MAC protocol to prevent packet collisions, such as listen-before-talk and back-off mechanisms, packets might still collide and lead to unbounded channel access delays, especially under heavily loaded channel conditions [2], [4], [7], [8]. This shortcoming makes CSMA a questionable choice for platooning applications, where high timing and reliability requirements combined with a high beacon update rate present particularly challenging MAC conditions.

Improving communication delay and reliability has already been studied several times by the VANET research community, and some methods have been proposed to tackle these issues, e.g., [3], [9], [10]. Although these proposals manage to keep the channel access delay and packet loss at acceptable levels, most of them are designed to obtain benefits for generic vehicular ad hoc networks, and not for specific applications such as platooning. Some works focus on practical issues and their impacts on platooning performance, such as [11] and [12]. In [11] the impact of packet loss on the performance of platooning applications was evaluated, and in [12] antenna placement and its impact on the packet error rate is evaluated using real-world experiments. However, most research about platooning is related to strategies for improving timing and reliability either through the introduction of TDMA slots, [2], [3], [7], [10], [13] or through retransmissions [4], [5]. Luckily [8] also uses retransmissions.

However, TDMA based schemes typically require slot synchronization and they are not very dynamic when it comes to changing the beacon period or scheduling retransmissions. Similarly, schemes including retransmissions usually requires extra overhead for control data and scheduling and also a centralized control unit to determine when and if retransmissions are needed. In contrast, our token-based method does not require synchronization nor extra overhead for scheduling or control traffic. Moreover, it is decentralized, adapts easily to changes in the beacon frequency and the amount of redundancy introduced through retransmissions can be adapted based on instantaneous conditions.

\section{TOKEN-BASED MAC PROTOCOL}

Assume that a platoon is composed of a leading vehicle and one or more regular platoon members following the leader, each one broadcasting status updates (beacons). In each platoon, we assume that there is a token manager acting as a central controller, the token manager, preferably located in the middle of the platoon, offering the best connectivity to other platoon members. This assumption is not unreasonable given that a platoon is limited due to practical constraints, such as avoiding to block highway exits. We also assume that the information about who is the token manager is available to all platoon members. Since all beacons are broadcasted, it enables piggybacking the token on each transmitted beacon. This implies that for each beacon, all nodes are notified about which node is given the token, and thereby is allowed to access the channel next. The term token refers to a privilege, given to a platoon member when it is chosen by the current token holder, to be the next one to transmit the beacon. Whenever a platoon member is given the token, it will transmit its beacon as soon as the channel is sensed free and at the same time, it will pass on the token to the next token holder. Note that this method does not require any extra packet transmissions for token passing.

To select the next token holder, each node must create a list of all platoon members and keep it updated based on all received beacons. Whenever a platoon member receives the token, it selects the platoon member in its list which has the highest (data) age as the next vehicle to transmit its beacon. We assume that a beacon is not discarded until a new one (with fresher information) is available. This means that a beacon is available for re-broadcasting within its beacon interval whenever the vehicle gets the token. Due to the data age based selection of the next token holder, vehicles, that have not been successful in broadcasting their beacon, will be prioritized and get retransmission opportunities that considerably increase the probability of successful beacon reception.

After receiving a beacon with the piggybacked token, the newly appointed token holder must wait for a predefined period of time, $t_{T H N}$, (except for the token manager that requires a longer waiting time as explained below) which is a function of the propagation time from the first to the last vehicle in the platoon, before it can begin its beacon transmission. This way, we can be sure that no simultaneous transmissions take place, thereby avoiding collisions.

There are two main issues that need to be considered in token-based scheduling, especially when packet losses due to unstable channel conditions are to be expected. First, recovering from a lost token, and second, (re-)joining disconnected or new members into the platoon. Considering the characteristics of the channel in vehicular ad-hoc networks, tokens may be lost due to an unreliable wireless channel. We therefore define two different token management tasks: 
(a) For the token manager: the token manager is the one responsible to generate the first token. If a beacon is lost due to connectivity issues the token will also be lost as it is piggybacked on the beacon itself. In case of packet loss, the token manager must re-generate the token by (re-)broadcasting its beacon and selecting a new member as the next token holder. The token manager therefore monitors the channel and, if it cannot detect any beacon transmission for a pre-defined period of time equal to three times the propagation time, i.e., $t_{R G}=$ $3 t_{T H N}$, it will generate a new token for a platoon member selected based on the age in its local list. The parameter $t_{R G}$ is maximum time between two constitutive beacon receptions and includes one propagation time for the token to be passed on plus one propagation time since the token holder needs to defer its transmission, and finally one more propagation time for the new token to be received by the token manager. In order to avoid a ping-pong effect between the token manager and a distant platoon member in outage, the token manager has to select a new token holder from its local list each time the token is lost. Since this list is ordered by beacon age, the new selected member is the one with the second highest data age.

(b) For other platoon members: the only responsibility is to select the next token holder every time it receives the token, and to announce it to all platoon members through a beacon transmission.

\section{A. Temporary disconnection}

As mentioned above, the token manager is located such that it should be able to receive all beacon transmissions sent by other platoon members. However, as the platoon length increases, the probability of not being able to hear all platoon members all the time also increases. We consider the case when a transmission from one platoon member cannot be directly received by another, very distant platoon member. In this case, whenever a member fails to receive a beacon from a distant member during one entire beacon period, it removes the member from its local list of platoon members. Note, however, that the removed member would not be removed from the local lists of all members, and thus it will eventually be chosen as the next token holder by a vehicle close by and thereby it remains in the token loop.

A more serious problem occurs when one member is removed from the local lists of all other platoon members, including the token manager. In that rare but theoretically possible case, the removed member will be totally disconnected from the platoon, and it will not be given the token; thus, it has no chance to transmit its beacons. In order to allow totally disconnected members to again join the platoon and receive the token, the token manager will, each time it receives the token, wait for a period longer than the rest of the platoon members, namely $t_{T M}$, thereby allowing disconnected members to join the platoon. The value of $t_{T M}$ is calculated according to:

$$
t_{T M}=t_{T H N}+t_{J}
$$

where $t_{J}$ is extra time needed to allow disconnected members to re-join the platoon, or even new members to join it. The value of $t_{J}$ depends on the propagation time, the beacon packet length and the maximum back-off time. A new member joins by broadcasting its beacon during $t_{J}$, competing for channel access according to the IEEE 802.11p-compliant CSMA random access protocol. As soon as new members are notified that the token manager is selected as the next token holder, a random back-off counter is initiated by all nodes attempting to join the platoon. When the token manager receives the token, it defers its transmission and allows the next token holder is chosen by competition between all nodes attempting to join the platoon. The newly joined member must give the token directly to the token manager by selecting it as the next token holder. The token manager resumes and transmits its beacon together with a new token holder selected due to having the highest age in its local list. A platoon member that recognizes that the token is passing but it was not selected as a token holder for an entire beacon period must switch to the re-join state and waits for a re-join period to again join the platoon. The re-join phase can also be extended to even accommodate possible event-based emergency messages.

\section{B. Built-in retransmission scheme}

In the IEEE 802.11p standard [6] there is no retransmission scheme for unsuccessful broadcast transmissions since there is no way to determine if reception was successful. Simply increasing the beacon update rate might just add to the problem by increasing the probability of packet collisions and lower the performance even further [4]. Our method actually proposes a built-in retransmission scheme as platoon members search in their lists and select a member based on beacon age to pass the token to. The algorithm just keeps selecting the nodes with the highest data age, thereby automatically offering retransmission opportunities to those nodes that had no success for a while. This way, the transmitter side does not need any mechanisms such as acknowledgements, to guarantee a successful reception at the receiver side. Therefore, the number of retransmissions is dynamically selected based on the current channel condition. Also, a certain maximum time allowed for retransmissions in each beacon interval can be set, by preventing the token manager from initiating another round of retransmissions after a certain time.

Our protocol also introduce a more flexible and scalable scheduling mechanism compared to TDMA-based schemes for VANETs and specifically for platooning applications. Due to the distributed nature of the protocol, members independently manage beacon transmissions and, as a result, no rescheduling is needed for changes, and the protocol is able to automatically adapt itself to changes in the network scenario such as platoon size or beacon generation frequency. A pre-scheduled TDMAbased retransmission scheme on the other hand is much more static and requires rescheduling and control data exchange to adapt to changes.

\section{Simulation Details}

To assess the effectiveness of the proposed protocol we rely on computer simulations. In this section, we describe the simulation details, including simulation scenario parameters and protocol configurations.

We simulate platoons of five or ten vehicles on a highway, with an antenna-to-antenna spacing of $30 \mathrm{~m}$. We used SUMO 
[14] in order to generate realistic vehicular mobility models. Also, we implemented our proposed MAC protocol in OM$\mathrm{NeT}++$ (version 4.4.1) [15], and used the IEEE 802.11p implementation made available by the Veins framework (version 2.1) [16] for OMNeT++ for performance comparison purposes. Table I summarizes the simulation parameters.

TABLE I. THE SIMULATION PARAMETERS

\begin{tabular}{ll}
\hline Simulation Parameter & Value \\
\hline \hline Simulation time & $20 \mathrm{~s}$ \\
Platoon size & 5 or 10 vehicles \\
Transmission range & $500 \mathrm{~m}$ \\
Propagation model & Simple path loss \\
& + Log-normal shadowing \\
Beacon frequency & 10,50 and $100 \mathrm{~Hz}$ \\
Packet length & $400 \mathrm{bytes}$ \\
Frequency & $5.9 \mathrm{GHz}$ \\
Data Rate & $6 \mathrm{Mbps}$ \\
Antenna-antenna spacing & $30 \mathrm{~m}$ \\
$t_{T H N}$ & $0.4 \mathrm{~ms}$ \\
$t_{T M}$ & $1.2 \mathrm{~s}$ \\
$t_{J}$ & $0.3 \mathrm{~ms}$ \\
\hline \hline
\end{tabular}

Similarly to the study in [17], we combine simple path loss and log-normal shadowing models, which are common models for highway simulation. We have chosen Inter-Reception Time (IRT) as the performance metric; it is calculated as the time interval between the sequential reception of beacons from each member averaged over all platoon members. The IRT parameter reflects the data age of the beacon content as it monitors the age of the information a node holds from a specific neighbor once a new beacon arrives. Maintaining a IRT close to the beacon period is vital to the successful implementation of a platoon control application.

\section{Simulation RESUlts And AnAlysis}

In order to quantitatively evaluate the proposed mechanism, our protocol is compared to IEEE 802.11p for different numbers of vehicles and beacon frequencies. For this purpose, we determine the beacon inter-reception time to show the efficiency of the system in reception of safety messages within time constraints, as well as the reliability of beacon delivery.

For beacon frequency of $50 \mathrm{~Hz}$ and a platoon with 5 vehicles, as seen in Figure 1, our protocol clearly outperforms IEEE $802.11 \mathrm{p}$. The figure shows that our protocol keeps an IRT below $20 \mathrm{~ms}$ which means it can deliver all beacons before the next beacon generation. In this case, the built-in retransmission scheme of our protocol has a fundamental role to achieve these results since each beacon gets a chance to be broadcasted depending on the current channel situation. As all intra-platoon communication takes place on a dedicated service channel, we can fully utilize the available bandwidth without interfering with the performance of other VANET applications. Also, it allows us to support a higher-frequency beacon generation for safety applications requiring very high beacon generation frequency. In IEEE $802.11 p$, on the other hand, since all vehicles try to access to the channel in a short time window after each beacon generation time, and since there is no retransmission opportunity, there is a high probability of collision which causes beacon interval times of more than $20 \mathrm{~ms}$. Moreover, as shown in the figure, the longest interreception time for IEEE $802.11 \mathrm{p}$ amounts to $120 \mathrm{~ms}$, rendering vehicles invisible to their neighbors for large time periods due to repeated beacon collisions and thereby endangering the safe operation of the platoon.

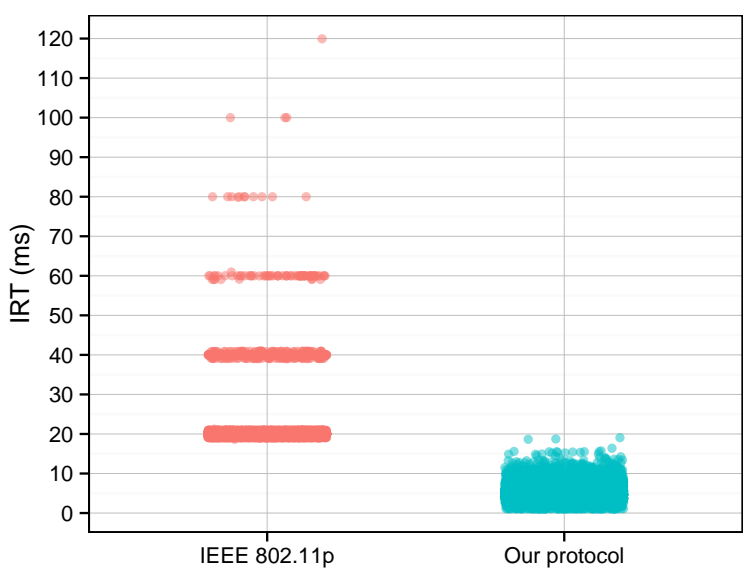

Fig. 1. IRT for a platoon with 5 vehicles and beacon frequency of $50 \mathrm{~Hz}$.

In Figure 2, we evaluate the performance of our protocol for two different platoon sizes of 5 and 10 vehicles, when the beacon frequency is equal to $50 \mathrm{~Hz}$. Complementing Figure 1 , this figure shows that when the platoon size is increased to 10 vehicles, our protocol cannot maintain the IRT below one beacon generation interval. However, it still can keep the maximum IRT below three beacon generation intervals, and deliver $97 \%$ of the beacons before $20 \mathrm{~ms}$, while the maximum IRT obtained for IEEE 802.11p is equal to $300 \mathrm{~ms}$.

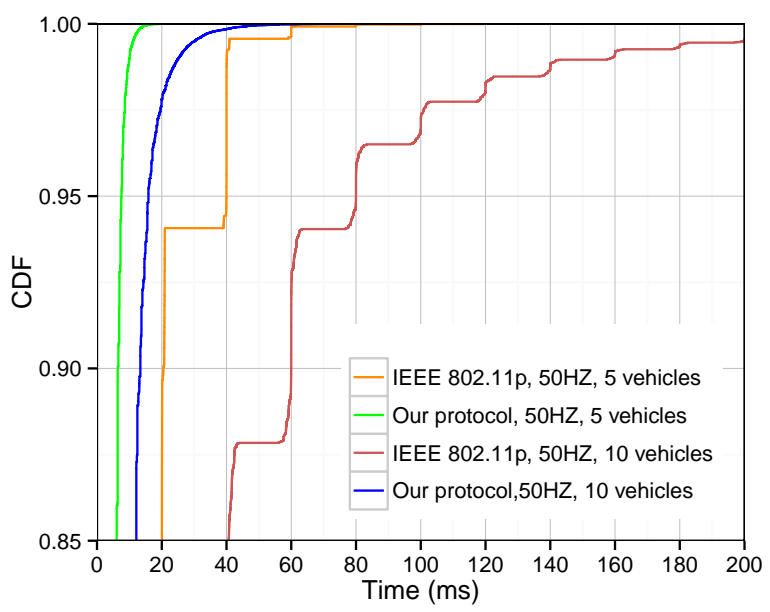

Fig. 2. IRT for beacon frequency of $50 \mathrm{~Hz}$.

A beacon frequency of $50 \mathrm{~Hz}$ is often mentioned by truck manufacturers as the target frequency for platooning applications. In order to evaluate the performance of our protocol under more challenging conditions we increased the beacon frequency to $100 \mathrm{~Hz}$. As shown in Figure 3, when increasing the beacon frequency, the IRT also increases so that, if $92 \%$ of inter-reception times for IEEE 802.11p, when the beacon frequency is $50 \mathrm{~Hz}$, are less than three beacon generation intervals, for a beacon frequency of $100 \mathrm{~Hz}$ this number goes 
down to $85 \%$. Since our protocol keeps the token circulating, a received token is used as a retransmission opportunity at low beacon frequencies whenever there is no new beacon. With increasing beacon frequency, however, the beacon generation interval decreases and the number of retransmission opportunities is also decreased. Therefore, the available bandwidth is instead used for broadcasting new beacons, but we still obtain performance improvements also with fewer retransmissions.

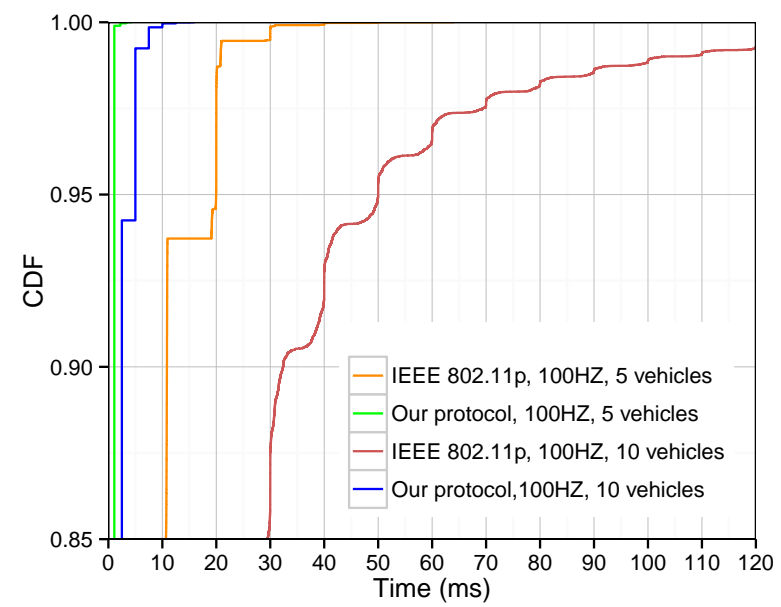

Fig. 3. IRT for beacon frequency of $100 \mathrm{~Hz}$.

\section{CONCLUSION}

In this paper, we propose a token-based medium access mechanism which is able to transmit beacons within time constraints more reliably than IEEE 802.11 p. The proposed mechanism uses a token for exclusive access to the channel and keeps requesting retransmissions from nodes based on data age, thereby decreasing number of collisions and also automatically offering retransmission opportunities to those nodes that had no success for a while. Simulation results show that the method is able to decrease the beacon inter-reception time and guaranteeing beacon delivery within one beacon generation interval for short platoons, which are very critical requirements for the safe maintenance of a platoon travelling at short inter-vehicle distances. Also, it clearly outperforms IEEE 802.11p in several different scenarios where the platoon size and beacon frequency changes. In addition, our token-based method is decentralized and does not require synchronization or any extra overhead for control traffic. It adapts easily to changes in the beacon frequency and the number of platoon members. If constraints on channel busy ratio are imposed, the amount of retransmissions can be limited by preventing the token manager from initiating another round of retransmissions after a certain time. Also note that event-driven messages can be initiated each time the token manager holds the token.

\section{ACKNOWLEDGMENT}

This work was partially supported by the Ministerio de Ciencia e Innovación, Spain, under Grant TIN2011-27543C03-01. Böhm and Uhlemann are funded by the Knowledge Foundation through the ACDC project.

\section{REFERENCES}

[1] C. Bonnet and H. Fritz, "Fuel consumption reduction in a platoon: Experimental results with two electronically coupled trucks at close spacing," SAE International, 2000.

[2] K. Bilstrup, E. Uhlemann, E. Strom, and U. Bilstrup, "Evaluation of the IEEE 802.11p MAC method for vehicle-to-vehicle communication," in Proc. IEEE VTC2008-Fall, Sep 2008, pp. 1-5.

[3] H. Omar, W. Zhuang, and L. Li, "VeMAC: A TDMA-based MAC protocol for reliable broadcast in VANETs," IEEE Trans. Mobile Computing, vol. 12, no. 9, pp. 1724-1736, Sept 2013.

[4] A. Böhm, M. Jonsson, and E. Uhlemann, "Performance comparison of a platooning application using the IEEE 802.11p MAC on the control channel and a centralized MAC on a service channel," in Proc. WiMob, Oct 2013, pp. 545-552.

[5] A. Böhm and K. Kunert, "Data age based retransmission scheme for realiable control data exchange in platooning applications," in to appear in Proc. ICC Workshops 2015, Jun 2015.

[6] IEEE Std 802.11-2012, "IEEE Standard for Information technology - Telecommunications and information exchange between systems Local and metropolitan area networks - Specific requirements - Part 11: Wireless LAN Medium Access Control (MAC) and Physical Layer (PHY) Specifications," 2012.

[7] M. Segata, B. Bloessl, S. Joerer, C. Sommer, M. Gerla, R. Cigno, and F. Dressler, "Towards inter-vehicle communication strategies for platooning support," in Proc. Nets4Cars-Fall, Oct 2014, pp. 1-6.

[8] B. Hassanabadi and S. Valaee, "Reliable periodic safety message broadcasting in VANETs using network coding," IEEE Trans. Wireless Communications, vol. 13, no. 3, pp. 1284-1297, Mar 2014.

[9] Y.-C. Lai, P. Lin, W. Liao, and C.-M. Chen, "A region-based clustering mechanism for channel access in vehicular ad hoc networks," IEEE JSAC, vol. 29, no. 1, pp. 83-93, Jan 2011.

[10] A. Alonso and C. Mecklenbrauker, "Stabilization time comparison of CSMA and self-organizing TDMA for different channel loads in VANETs," in Proc. ITST, Nov 2012, pp. 300-305.

[11] C. Lei, E. van Eenennaam, W. Wolterink, G. Karagiannis, G. Heijenk, and J. Ploeg, "Impact of packet loss on CACC string stability performance," in Proc. ITST, Aug 2011, pp. 381-386.

[12] C. Bergenhem, E. Hedin, and D. Skarin, "Vehicle-to-vehicle communication for a platooning system," Procedia - Social and Behavioral Sciences, vol. 48, no. 0, pp. 1222 - 1233, 2012, Transport Research Arena 2012.

[13] P. Fernandes and U. Nunes, "Platooning with IVC-enabled autonomous vehicles: strategies to mitigate communication delays, improve safety and traffic flow," IEEE Trans. ITS, vol. 13, no. 1, pp. 91-106, Mar 2012.

[14] L. E. J. Behrisch, M. Bieker and D. Krajzewicz, "SUMO - Simulation of Urban MObility: an overview in: SIMUL 2011," in The Third International Conference on Advances in System Simulation, 2011.

[15] “OMNeT++ home page," http://www.omnetpp.org, accessed: 2014-0230 .

[16] C. Sommer, R. German, and F. Dressler, "Bidirectionally coupled network and road traffic simulation for improved IVC analysis," IEEE Trans. Mobile Computing, vol. 10, no. 1, pp. 3-15, Jan 2011.

[17] N. Akhtar, S. Ergen, and O. Ozkasap, "Vehicle mobility and communication channel models for realistic and efficient highway VANET simulation," IEEE Trans. Vehicular Technology, vol. 64, no. 1, pp. 248262, Jan 2015 